\title{
Metodología para estimar y evaluar un modelo de gestión del conocimiento mediante ecuaciones estructurales
}

\author{
A methodology for estimating and evaluating a knowledge \\ management model using structural equations
}

\section{Metodologia para estimar e avaliar um modelo de gestão do conhecimento usando equações estruturais}

\author{
Milena Zabaleta-de Armas ${ }^{1}$, Luís E. Brito-Carrillo², Manuel A. Garzón-Castrillón ${ }^{3}$
}

\footnotetext{
1 Cont. Publi, MSc, profesor e investigador Grupo de investigación Pensamiento Contable, Universidad Simón Bolívar

2 Ing. Sist, MSc, coordinador de Soporte Tecnológico, Universidad Simón Bolívar

3 Adm. Empre; MSc; PhD; Post PhD, Director Grupo de investigación FIDEE

Email: mzabaleta@unisimonbolivar.edu.co
}

\section{Resumen}

Este artículo presenta una metodología para la estimación y evaluación de un modelo de gestión del conocimiento, utilizando ecuaciones estructurales, que sirven para probar y estimar relaciones causales a partir de datos estadísticos y referentes teóricos sobre la causalidad, mostrando los indicadores más influyentes que se deben intervenir para mejorar la gestión del conocimiento en el área de TI de la universidad Simón en Barranquilla, con base en las variables TIC, Capital Intelectual, Aprendizaje Organizacional e Innovación. Logrando demostrar que el instrumento diseñado es confiable según los resultados de la prueba Alfa de Cronbach y útil para medir la Gestión del Conocimiento, en las variables más influyentes: Las TIC, servicios principales y de infraestructura; el capital intelectual, enfocado al capital estructural, clima organizacional y los procesos de negocios; el aprendizaje organizacional, en el Sistema cultural y el aprendizaje en equipo y la innovación, referida a políticas que motiven la creatividad del personal.

Palabras claves: Aprendizaje organizacional; Ecuaciones estructurales; Gestión del conocimiento; instrumento de investigación

\footnotetext{
Abstract

This article describes a structural equation-based methodology for estimating and evaluating a knowledge management model for testing and estimating causal relationships from statistical data and theoretical references on causality. It highlights the most influential indicators that should be considered for improving knowledge management in the IT area of the Simón University in Barranquilla, based on ICT, intellectual capital, organisational learning and innovation. It demonstrates that the 
designed instrument was reliable according to Cronbach's alpha test and was useful for measuring knowledge management regarding the most influential variables. These were ICT (main services and infrastructure), intellectual capital (focusing on structural capital, organisational climate and business processes), organisational learning (cultural system and team learning) and innovation (referring to policies encouraging personal creativity).

Keywords: organisational learning; structural equation; knowledge management; research instrument.

\section{Resumo}

Este artigo apresenta uma metodologia para estimar e avaliar um modelo de gestão do conhecimento, utilizando equações estruturais, que servem para testar e estimar relações causais a partir de dados estatísticos e referências teóricas sobre causalidade, mostrando os indicadores mais influentes que devem ser intervir para melhorar a gestão do conhecimento na área de (Tecnologia e inovação) TI da Universidade Simon Bolívar em Barranquilla, com base nas variáveis TIC: Capital Intelectual, Aprendizagem Organizacional e Inovação. Logrando demonstrar que o instrumento projetado é confiável de acordo com os resultados do teste Alpha de Cronbach que e útil para medir a Gestão do Conhecimento, nas variáveis mais influentes: as TIC, principais serviços e infraestrutura; capital intelectual, focado em capital estrutural, clima organizacional e processos de negócios; Aprendizado organizacional, no sistema cultural e aprendizado e inovação em equipe, referentes a políticas que estimulam a criatividade pessoal.

Palavras-chave: Aprendizagem organizacional; Equações estruturais; Gestão do conhecimento; instrumento de pesquisa

Clasificación JEL. M15, M19

\section{Introducción}

En cada organización donde se ha puesto en marcha la gestión del conocimiento, se hace de una manera particular tanto en el área organizacional, cultural o en lo tecnológico, por lo que no existe un modelo estándar, lo que motivo crear uno propio acorde a las necesidades del área de TI de la universidad, ya que gran parte del conocimiento reside en las personas; es por esto que cuando se van de las organizaciones, se lo llevan, por lo que es de vital importancia, crear el ambiente necesario, para propiciar la adquisición, creación, difusión y aplicación del mismo; convirtiendo la gestión del conocimiento en una prioridad para los directivos, identificando el conocimiento tácito disperso en las personas con el fin de sintetizar dicho conocimiento e incorporarlo en las actividades claves de la organización.

Por lo anterior se hace necesario realizar un análisis de las variables que integran la gestión del conocimiento y su interrelación, en este caso se ha diseñado un cuestionario y se ha utilizado la estimación y evaluación de un modelo utilizando las ecuaciones estructurales. La gran ventaja de este tipo de modelos es que permiten proponer el tipo y dirección de las relaciones que se espera encontrar entre las diversas variables contenidas en él, para pasar posteriormente a estimar los parámetros que vienen especificados por las relaciones propuestas a nivel teórico. Por este motivo se denominan también modelos confirmatorios, ya que el interés fundamental es "confirmar" mediante el análisis de la muestra las relaciones propuestas a partir de la teoría explicativa que se haya decidido utilizar como referencia.

Por estas razones, este artículo se organiza como sigue: un marco analítico; instrumento de la investigación, gestión del conocimiento y estrategias de gestión del conocimiento; variables de análisis para la gestión del conocimiento; modelos de ecuaciones estructurales; metodología; resultados; fiabilidad; hipótesis estadística; prueba de fiabilidad de alfa de cronbach por variable de ecuaciones estructurales inicial; modelo de ecuaciones estructurales inicial; modelo final propuesto; descripción del modelo; modelo estructural; modelo de medida; ajuste del modelo de medida; ajuste del modelo estructural y conclusiones.

\section{Estado actual del conocimiento}

\section{Gestión del conocimiento}

Las definiciones de gestión del conocimiento son tan diversas algunas hablan de procesos, actividades, utilidad práctica, pero en general giran en torno a tres corrientes tal como lo referencian Seaton y Bresó (2001): la corriente organizacional, la cual valora los aportes para el proceso de aprendizaje y desarrollo organizacional. En esta corriente se destacan los aportes de Drucker (2000), Nonaka (1999) y Garvin (1993) y la corriente económica, valora en mayor grado la capacidad de generar dinero del conocimiento. Aquí se destacan los aportes de Bukowitz y Williams (1999), Klasson (1999) y Tiwana (2000) y la tercera corriente que contempla tanto lo económico como lo organiza- 
cional Garzón y Fisher (2008); Barragán (2009); (Prieto, 2003); Alvarado (2009), Kalpič y Bernus (2006), Schulze (2003)

Se concibe por parte de los autores, la gestión del conocimiento como una herramienta que permite implementar la estrategia competitiva de una organización, gobernando un proceso que consiste en: Identificar, adquirir, almacenar, difundir, compartir, utilizar y actualizar el conocimiento tácito y explícito. Convirtiéndose así en una herramienta de aprendizaje y en un catalizador para la innovación, que combinado con una adecuada estrategia de negocio proporciona ventaja competitiva. Basados en Alvarado et al., (2009); Kalpič y Bernus (2006), Schulze (2003), Garzón y Fisher (2008) y Barragán (2009), Zabaleta, et al., (2016)

\section{Variables de Análisis para la Gestión del Conocimiento}

Como resultado de una investigación realizada por los investigadores en el área de TIC de una universidad del caribe colombiano se determinó que las variables más influyentes para la gestión del conocimiento son: las tecnologías de la información y la comunicación TIC, el capital intelectual, el aprendizaje organizacional y la innovación; Zabaleta, et al., (2016)

\section{Tecnologías de la información y la comunicación TIC}

Infraestructura tecnológica, representada en hardware, software, redes de comunicación y procesos; que, asociadas a unas metodologías de uso o mejores prácticas, soportan y potencian el crecimiento de la organización. De Vita (2008), Thompson y Strickland (2004), Tseng (2008), O’Brien y Marakas (2006)

Para poder hablar de los diversos tipos de herramientas que hoy se encuentran en el mercado para apoyar la gestión del conocimiento, resulta muy práctico hacer algún tipo de clasificación, teniendo en cuenta las diversas tareas y los procesos que se llevan a cabo durante la gestión del conocimiento, así como la infraestructura que las soporta. Para tal fin se toma de (Housel y Bell, 2001) la siguiente clasificación:

\section{Herramientas TIC de los servicios de infraestructura}

Estas herramientas normalmente son necesarias para implementar cualquier solución de gestión del conocimiento, se clasifican en: Servicios de Comunicación, Servicios de Colaboración, Servicios de Traducción,
Servicios de gestión de flujos de trabajo, Intranet y extranet y Agentes inteligentes. Lozano y Rodríguez (2012)

\section{Herramientas TIC de los servicios principales}

Define las soluciones de gestión del conocimiento porque ellas acceden directamente los repositorios de conocimiento. Estos servicios se basan en los procesos de creación, organización, y utilización de los repositorios. Ocampo et al., (2009)

\section{Herramientas TIC de los servicios empaquetados}

Hacen referencia a servicios de bajo nivel que resuelven problemas de tipo específico, tales como la gestión de la relación con los clientes, se ha dedicado mucha atención en la literatura a este tipo de servicios porque tienen relación directa con los clientes ya que a los gerentes les resulta relativamente fácil justificar su compra. Lozano y Rodríguez (2012)

Toda la infraestructura tecnológica explicada anteriormente hace parte del capital estructural, que es un facilitador del flujo de conocimiento y una mejora en la eficacia de la organización, cuando este se acompaña con innovación, optimiza la gestión de los procesos de negocio utilizando las Tecnologías de la Información y las Comunicaciones ( $\mathrm{TIC}^{\prime} \mathrm{s}$ ) Cegarra, Alonso y Monreal (2006)

De lo expuesto anteriormente se propone la segunda hipótesis:

H2: El uso de las (TIC) produce un efecto positivo en el Capital Intelectual $(\mathrm{Cl})$ del departamento de sistemas y recursos tecnológicos de la Universidad.

\section{Capital Intelectual}

Corriente Estratégica que analiza la búsqueda, creación y uso eficaz del conocimiento organizativo que poseen las personas, la estructura organizativa y las relaciones internas y externa de una organización, para obtener ventaja competitiva sostenida y generar valor. Bueno, Salmador y Merino (2008).

Son muchas las clasificaciones que se le da al capital intelectual, el modelo más conocido y utilizado es el propuesto por Bontis, Keow y Richardson (2000) quienes lo dividen tres dimensiones: capital humano, estructural y relacional. Autores como Andriessen (2004), Choo y Bontis (2002) también realizan aportes significativos respecto a esta división. 
Dado que el aprendizaje organizacional inicia por el aprendizaje individual de cada integrante de la organización y ello se estudia dentro del capital humano, que autores como Bontis, Crossan y Hulland (2002), Bueno Campos (2000), Camison Zornosa, Palacios Marques y Devece Carañana (2000), Euroforum (1998), Ordoñez de Pablos $(2002,2003)$ y Petrash $(1996,2001)$ consideran que dicho capital se encuentra integrado por el stock de conocimiento tanto explícito y táctico que poseen las personas. Sánchez (2004) indica que el capital humano es el valor que reside en los miembros de la organización y permite generar también valor a la empresa. Kaplan y Norton (2004) miden el capital humano atendiendo a las capacidades, la formación y el conocimiento. Roos et al., (2001) manifiesta que se encuentra compuesto por tres elementos: competencias (conocimientos, capacidades, talento y know how); Bontis et al., (2002) indica que adicionalmente se compone de elementos como la actitud (conducta, motivación, actuación y ética) y agilidad intelectual.

De lo anterior se propone la tercera hipótesis:

H3: El Capital Intelectual $(\mathrm{Cl})$ produce un efecto positivo en el Aprendizaje Organizacional (AO) del departamento de sistemas y recursos tecnológicos de la Universidad.

El Capital estructural es un facilitador del flujo de conocimiento y una mejora en la eficacia de la organización, cuando este se acompaña con innovación, mejora la gestión de los procesos de negocio utilizando las Tecnologías de la Información y las Comunicaciones (TIC's). Cegarra, Alonso y Monreal (2006)

\section{Aprendizaje Organizacional}

Es la capacidad que tiene la organización para aprender, utilizando información de sus fuentes, para generar nuevo conocimiento en los sujetos del aprendizaje, propiciando la cultura y las condiciones para desarroIlar nuevas capacidades que apoyen la sustentabilidad. Nonaka y Takeuchi (1999) y Garzón y Fisher (2008) y de no desarrollarlas impiden que se dé una respuesta proactiva a los clientes o usuarios. Gómez, Villarreal y Villarreal (2015).

Po su parte Garzón y Fisher (2008) indican que conocer la capacidad que tiene una organización de aprender permite saber: qué se conoce, dónde está y cómo se puede usar. Y Camargo et al., (2006) indican que el gran objetivo de los procesos del conocimiento es la innovación, ya que es algo que las organizaciones precisan para sobrevivir y para crecer.

De lo anterior podemos plantear la cuarta hipótesis:
H4: El Aprendizaje Organizacional (AO) impacta positivamente la Innovación (INNOV) en el departamento de sistemas y recursos tecnológicos de la Universidad Simón Bolívar Barranquilla.

\section{Innovación}

Camargo et al.,(2006) indican que el gran objetivo de los procesos del conocimiento es la innovación y la forma, intensidad y las condiciones en que se dan los procesos de innovación, por su parte Nelson y Winter (1982) y Rodríguez-Castellanos et al., (2011), Hoyos et al., (2009) y Future Trends Forum (2007) coinciden en indicar que los recursos, en términos de competencias y capacidades, no son suficientes, siendo necesario que vayan acompañados de una cultura organizacional (valores y creencias compartidas) que sea capaz de crear una atmósfera de trabajo adecuada que incite y motive a sus miembros a que se esfuercen por innovar y a que en la empresa exista un clima que estimule la creatividad.

\section{Modelos de ecuaciones estructurales}

El concepto de modelos o sistema de ecuaciones estructurales (SEM) se introdujo en la década de los 30's, por Wrigth $(1921,1934)$, desarrolló una forma de romper las correlaciones observadas en un sistema de ecuaciones que describían su hipótesis respecto a las relaciones causales. Las relaciones entre variables fueron representadas en un "path diagram", por lo que su método llegó a conocerse como "path analysis" (Wrigth 1921, 1934). Años más tarde, este método fue redescubierto y desarrollado por economistas y sociólogos, de entre los cuales, los trabajos más sobresalientes son los de Jöreskog (1973,1977 y 1981) y Jöreskog y Sörbom (1982). En ellos, transformaron el "path analysis" de Wright en un nuevo método denominado "structural equation modelling", en el que se combina el análisis factorial con el path analysis." Wrigth $(1921,1934)$ Jöreskog $(1973,1977$ y 1981) y Jöreskog y Sörbom (1982) citados por (García, 2011).

Los modelos de ecuaciones estructurales para (García, 2011) analizan las relaciones causales entre datos directamente observables, la existencia de relación causal entre dos variables implica la existencia de correlación, asumiendo que hay un mecanismo subyacente que lleva a una estructura de covarianzas teóricas entre un vector de variables aleatorias, para diseñar y probar un modelo que tenga la esencia de este mecanismo subyacente y además son usados para validar modelos teóricos (Dávila, et al., 2012).

Para tal fin, hay que verificar primero si se cumplen los supuestos de normalidad y unidimensionalidad de las 
variables, para lograr la unidimensionalidad se deben eliminar los ítems necesarios para lograr que todos se encuentren en un solo factor, después de la eliminación de los ítems, es necesario realizar nuevamente la fiabilidad de los datos esto se puede realizar mediante análisis exploratorio. Escobedo, Hernández, Estebané, y Martínez, (2016).

En general los SEM permiten, establecer el grado en que las variables se pueden medir, después, incorpora constructos abstractos e inobservables (variables latentes y variables teóricas no observadas) y modela las relaciones entre múltiples variables predic-toras (independientes o exógenas) y variables criterio (dependientes o endógenas). Combina y confronta el conocimiento a priori e hipótesis con datos empíricos, por lo que los modelos de ecuaciones estructurales son más confirmatorios que exploratorios Haenlein $M$, Kaplan A. (2004) Cupani, (2012)

Los principales especialistas en el SEM consideran seis pasos a seguir para aplicar esta técnica: especificación, identificación, estimación de parámetros, evaluación del ajuste, re-especificación del modelo e interpretación de resultados (Kaplan, 2000; Kline, 2005). Además, incluyen un apartado a considerar: el análisis de la matriz de datos.

De lo anteriormente expuesto se plantea la quinta y última hipótesis:

H5: Las variables Aprendizaje Organizacional, TIC, Capital Intelectual e Innovación que conforman el modelo de gestión del conocimiento propuesto están correlacionadas.

\section{Materiales y métodos}

Se realizó un estudio correlacional, para determinar la incidencia de un modelo de gestión del conocimiento en el aprendizaje y aplicación de los procesos llevados a cabo en el área de soporte tecnológico de la Universidad Simón Bolívar.

\section{Instrumento de la investigación}

Hay cuatro criterios que garantizan el rigor, autenticidad y validez del proceso de investigación según Lincoln y Guba (1985): veracidad, aplicabilidad, consistencia y neutralidad. Dichos criterios guardan estrecha relación con los instrumentos utilizados.

Como la realidad de cada organización es única, la calidad de una investigación depende de la calidad de los datos utilizados y éstos dependerán del instrumento con que se hayan recolectado Rodrigo A. (2006).
De esta manera la construcción de un cuestionario exige una actividad técnica, por parte del investigador. Para realizar la fiabilidad del instrumento diseñado se utilizó el estadístico Alfa de Cronbach, el cual mide la consistencia interna de cada escala. Una escala será fiable cuando más cercano esté el valor del Alfa de Cronbach a 1 , se considera que el instrumento de medida es adecuado con un valor del Alfa de Cronbach mayor o igual que 0,70. Asún (2006)

Con base en lo anterior se propone una metodología que incluye la construcción de un cuestionario, se platea la primera hipótesis

H1: Las escalas de medida utilizadas en el cuestionario para conocer el estado de la gestión del conocimiento del departamento de sistemas y recursos tecnológicos de la Universidad son fiables.

Un amplio sector de investigación de la gestión empresarial de los últimos años ha utilizado el modelo de ecuaciones estructurales (SEM) como un enfoque analítico que combina simultáneamente modelos de análisis factorial y de regresión lineal para la comprobación de las hipótesis teóricas. Bernal, de Nieves y Briones, A. (2016); León y Fernández (2018); Salcines y González (2015).

\section{Hipótesis Estadísticas}

Antes de realizar el análisis confirmatorio de fiabilidad y dimensionalidad mediante las técnicas de Modelos de Ecuaciones Estructurales se definen y plantean las hipótesis que se quieren confirmar en este artículo. Batista-Foguet, Coenders y Alonso, (2004), El proceso de validación de un cuestionario implica un conjunto de decisiones que se apoyan en contrastes de hipótesis correctamente formuladas.

Se plantearon cinco (5) hipótesis en el marco analítico para validar el modelo propuesto, teniendo en cuenta a Abreu (2012) quien plantea que generalmente cuando se presentan variables de estudio complejas, se hace necesario o adecuado especificar dimensiones de estudio y posteriormente, establecer los indicadores".

Para el diseño del instrumento se construyeron cuadros metodológicos para cada una de las variables, estos cuadros se estructuraron como aparece en la Tabla 1.

Basados en esta estructura fue diseñado el instrumento inicial, que tuvo un total de 199 ítems. El instrumento fue valorado por dos expertos, realizaron correcciones a la redacción de algunos ítems y eliminaron otros. 
Tabla 1. Cuadro Metodológico Usado

\begin{tabular}{|c|c|c|}
\hline \multicolumn{3}{|c|}{ Nombre de la Variable } \\
\hline Definición Conceptual: & Definición Operativa: \\
\hline Dimensiones & Indicador & Ítems/ Afirmaciones \\
\hline
\end{tabular}

Fuente: Cazau (2006), Argyrous (2011), Abreu (2012).

Tabla 2. Análisis Demográfico Prueba Piloto

\begin{tabular}{|c|c|c|c|c|c|}
\hline Edad & Número & Disciplina & Formación & Por especialidad & Ciudad \\
\hline \multirow{2}{*}{$15-20$} & \multirow{2}{*}{2} & \multirow{2}{*}{2} & \multirow{2}{*}{ Especialista } & 1 & Medellín \\
\hline & & & & 1 & Cúcuta \\
\hline \multirow{6}{*}{$21-26$} & \multirow{6}{*}{20} & \multirow{3}{*}{12} & \multirow{3}{*}{ Profesional } & 2 & Medellín \\
\hline & & & & 1 & Cúcuta \\
\hline & & & & 9 & Barranquilla \\
\hline & & \multirow{2}{*}{7} & \multirow{2}{*}{ Especialista } & 6 & Barranquilla \\
\hline & & & & 1 & Cúcuta \\
\hline & & 1 & Maestría & 1 & Barranquilla \\
\hline \multirow{6}{*}{$27-32$} & \multirow{6}{*}{13} & \multirow{2}{*}{3} & \multirow{2}{*}{ Técnico } & 2 & Barranquilla \\
\hline & & & & 1 & Soledad \\
\hline & & 1 & Tecnológico & 1 & Barranquilla \\
\hline & & \multirow{3}{*}{9} & \multirow{3}{*}{ Profesional } & 2 & Soledad \\
\hline & & & & 1 & Puerto Colombia \\
\hline & & & & 6 & Barranquilla \\
\hline \multirow{2}{*}{$33-40$} & \multirow{2}{*}{6} & \multirow{2}{*}{6} & \multirow{2}{*}{ Tecnológico } & 1 & Soledad \\
\hline & & & & 5 & Barranquilla \\
\hline Mayor 40 & 1 & 1 & Doctorado & 1 & Barranquilla \\
\hline
\end{tabular}

Fuente: Elaboración propia.

Luego se realizó una prueba piloto con 192 ítems, tipo Likert auto administrado mediante google doc, la cual se aplicó a 42 funcionarios de TI de empresas que tuvieran un departamento o área de sistemas, el proceso de envío y respuesta demoró 25 días. Ver Tabla 2. Luego se eliminaron aquellos ítems con correlación de elementos-totales corregidos inferiores a 0.70. Se consideraron adecuados valores mayores o iguales 0.30 (Nunnally y Bernstein, 1995). Por lo que se eliminaron 98 ítems de los 192, quedando 94 ítems para ser analizados.

Después se realizó la reducción de dimensiones mediante el análisis factorial aplicando el método de extracción de las componentes principales determinándose la matriz de correlación mediante el análisis de KMO y prueba de esfericidad de Bartlett, visualizando la solución sin rotar y el gráfico de sedimentación, el tipo de rotación fue el de Equamax ya que esta es una combinación de los métodos de rotación
Varimax y Quartimax, simplificando los factores y las variables, minimizando tanto el número de variables que saturan altamente a un factor como el número de factores necesarios para explicar la variable, como resultado del anterior procedimiento quedaron 52 ítems para el nuevo instrumento aplicado a la población objeto de estudio, ver tabla 3. A los datos recogidos se le realizó un análisis factorial de componentes principales para eliminar ítems menos relevantes de cada variable, quedando 25 ítems, con los cuales se construye el modelo estructural inicial, al cual se le aplica un análisis confirmatorio para determinar la validez del modelo, y por último se ajusta utilizando índices determinados para tal efecto.

\section{Resultados}

Para llegar a los resultados que se describen a continuación, se utilizó la técnica de los modelos estructu- 
Tabla 3. Componentes Principales.

Variable aprendizaje organizacional

\begin{tabular}{|c|l|c|}
\hline Componente principal & \multicolumn{1}{|c|}{ Nombre del componente } & Afirmaciones o ítems \\
\hline 1 & $\begin{array}{l}\text { Condiciones para el aprendizaje con enfoque } \\
\text { en el compromiso de las directivas y de las } \\
\text { comunidades para aprender en equipo }\end{array}$ & $15,36,39,45,46,37,13,7$ \\
\hline 2 & $\begin{array}{l}\text { Competencias para aprender continuamente } \\
\text { utilizando la tecnología y el lugar } \\
\text { de trabajo para la formación }\end{array}$ & $34,42,23,17$ \\
\hline 3 & $\begin{array}{l}\text { Principios y valores que propician la discusión y el } \\
\text { cambio como resultado del aprendizaje colectivo }\end{array}$ & $5,43,49,32,27$ \\
\hline 4 & $\begin{array}{l}\text { Formación de competencias para la } \\
\text { Generación y uso del conocimiento }\end{array}$ & $1,52,40$ \\
\hline 5 & $\begin{array}{l}\text { Cultura humanista basada en los valores que } \\
\text { premia el aprendizaje y el trabajo en equipo }\end{array}$ & $30,48,50,47,51$ \\
\hline 6 & $\begin{array}{l}\text { El monitoreo permanente del cliente } \\
\text { como principal fuente de aprendizaje }\end{array}$ & $10,20,25$ \\
\hline
\end{tabular}

\section{Variable Tic}

\begin{tabular}{|c|l|c|}
\hline Componente principal & \multicolumn{1}{|c|}{ Nombre del componente } & Afirmaciones o ítems \\
\hline 1 & $\begin{array}{l}\text { Tic para aprender y gestionar de manera } \\
\text { integrada el conocimiento, enfocados } \\
\text { en su clasificación y utilización tanto del } \\
\text { conocimiento del negocio como del cliente. }\end{array}$ & $2,6,11,16,21,26,31,38$ \\
\hline
\end{tabular}

Variable capital intelectual

\begin{tabular}{|c|l|c|}
\hline Componente principal & \multicolumn{1}{|c|}{ Nombre del componente } & Afirmaciones o ítems \\
\hline 1 & Capital Estructural: Organización del conocimiento & 28 \\
\hline 2 & $\begin{array}{l}\text { Capital Estructural al servicio del capital humano: } \\
\text { Estructura organizativa, proceso y capacidades }\end{array}$ & $3,8,12$ \\
\hline 3 & Capital Estructural: Clima organizacional & $18,22,33$ \\
\hline 4 & Capital Estructural: Eficiencia de procesos & 41 \\
\hline
\end{tabular}

Variable innovación

\begin{tabular}{|c|l|c|}
\hline Componente principal & \multicolumn{1}{|c|}{ Nombre del componente } & Afirmaciones o ítems \\
\hline 1 & $\begin{array}{l}\text { Espíritu intraemprendedor: La comunicación } \\
\text { como inductor para generar y compartir } \\
\text { nuevos conocimientos e ideas. }\end{array}$ & $4,9,14,24,29$ \\
\hline 2 & $\begin{array}{l}\text { Espíritu intraemprendedor: Reconocimiento } \\
\text { como generador de Innovación }\end{array}$ & $19,35,44$ \\
\hline
\end{tabular}

Fuente: Elaboración propia.

rales SEM con el software estadístico Amos IBM SPSS 25. Para ajustar los modelos basándose en los indicadores para muestra pequeña ECIV y para la falta de normalidad el indicador PNFI, además se realizó un análisis con esta metodología para mejorar los indicadores GFI, AGFI, RMR y SRMR.

\section{Fiabilidad}

El primer examen de los datos es el estudio de la fiabilidad de las escalas de medida en cada una de las variables de estudio, (Innovacion, Aprendizaje Organizacional, Capital Intelectual y Tic, ver Tabla 4. 
Tabla 4. Resultado de Datos Cronbach.

\begin{tabular}{|c|c|c|}
\hline Variable & Alfa de Cronbach & No ítems \\
\hline $\mathrm{AO}$ & 0.97 & 28 \\
\hline $\mathrm{TIC}$ & 0.841 & 8 \\
\hline $\mathrm{Cl}$ & 0.819 & 8 \\
\hline Innovación & 0.914 & 8 \\
\hline
\end{tabular}

Fuente: Datos procesados con SPSS 25. Elaboración propia.

Análisis confirmatorio de fiabilidad y dimensionalidad aplicando la técnica de los modelos de ecuaciones estructurales

\section{Análisis Exploratorio de Unidimensionalidad}

Se realiza un análisis exploratorio de unidimensionalidad para cada una de las variables con el fin de confirmar si detrás de cada variable subyace un único factor, mediante un análisis factorial de componentes principales, con rotación Varimax a cada una de las variables, teniendo en cuenta que la unidimensionalidad de las escalas de medida se consideren satisfactoria cuando se extrae un solo factor (unidimensionalidad), con varianzas mayores a $60 \%$ y las cargas factoriales de cada ítem sean mayor a los 0.5 puntos.

\section{Prueba de fiabilidad de Alfa de Cronbach por variable}

Debido a que en todo este procedimiento se han eliminado 27 de las 52 variables observadas (Ítems) es necesario corroborar que se cumpla la fiabilidad de los datos por lo que se comprueba este supuesto nuevamente mediante el alfa de Cronbach, en donde se obtiene el valor más pequeño de 0.858 , por lo que los datos son altamente fiables. Los resultados de fiabilidad y de unidimensionalidad se resumen en la siguiente tabla 5 .

\section{Modelo de Ecuaciones Estructurales Inicial}

Tal y como ya se ha expuesto, el modelo de ecuaciones estructurales inicial se construye a partir de 25 variables observadas; variables que se corresponden con 25 ítems del cuestionario realizado, ver Figura 1.

A partir de ellas se crean 4 variables latentes diferentes a través de análisis factorial confirmatorio. Una vez creadas las variables latentes, se determinan las relaciones que éstas establecen entre sí, pudiendo ser relaciones de correlación y/o, relaciones de regresión.

En esencia la teoría establece que las variables latentes en estudio $\mathrm{AO}, \mathrm{TIC}, \mathrm{Cl}$ e INNOV, se encuentran correlacionadas entre sí por lo que se partirá bajo estas condiciones luego se ajustará el modelo y por último se confirmará de ser posible si los datos observados responden a lo que se expone en la teoría mediante la selección del mejor modelo.

Existen diversos programas informáticos para ajustar modelos: EQS, LISREL, AMOS, CALIS, etc. En la elaboración se ha utilizado el programa AMOS 22 por la posibilidad de trabajar con diagramas. La evaluación del modelo se ha efectuado utilizando diversos índices: NFI, GFI, AGFI, PNFI y RMSEA.

Tabla 5. Resultados de fiabilidad y de unidimensionalidad.

\begin{tabular}{|l|c|c|c|c|}
\hline \multicolumn{1}{|c|}{ Variable } & AO & TIC & CI & INNOV \\
\hline Alfa de Cronbach & 0.963 & 0.858 & 0.875 & 0.907 \\
\hline N $^{\circ}$ Ítems & 11 & 5 & 5 & 4 \\
\hline Cumple unidimensionalidad & $\mathrm{SI}$ & $\mathrm{SI}$ & $\mathrm{SI}$ & $\mathrm{SI}$ \\
\hline \% de la varianza & 74.861 & 64.656 & 70.17 & 78.428 \\
\hline Kolmogorov Smirnov & 0.202 & 0.204 & 0.192 & 0.183 \\
\hline Mediana & 6 & 6 & 5 & 5 \\
\hline Máximo & 7 & 7 & 7 & 7 \\
\hline MINIMO & 1 & 1 & 1 & 1 \\
\hline Asimetria & -0.761 & -0.877 & -0.42 & -0.413 \\
\hline Curtosis & -0.685 & -0.304 & -1.234 & -1.387 \\
\hline
\end{tabular}

Fuente: Datos procesados con SPSS 25. Elaboración propia. 
A continuación, se presenta la tabla 6, en donde se resumen los estadísticos de bondad de ajuste con su criterio de valor óptimo, no sin antes aclarar que estos valores de ajuste no son absolutos, se utilizarán como valores de referencia, ya que no hay un consenso definitivo al respecto. (García, 2011)

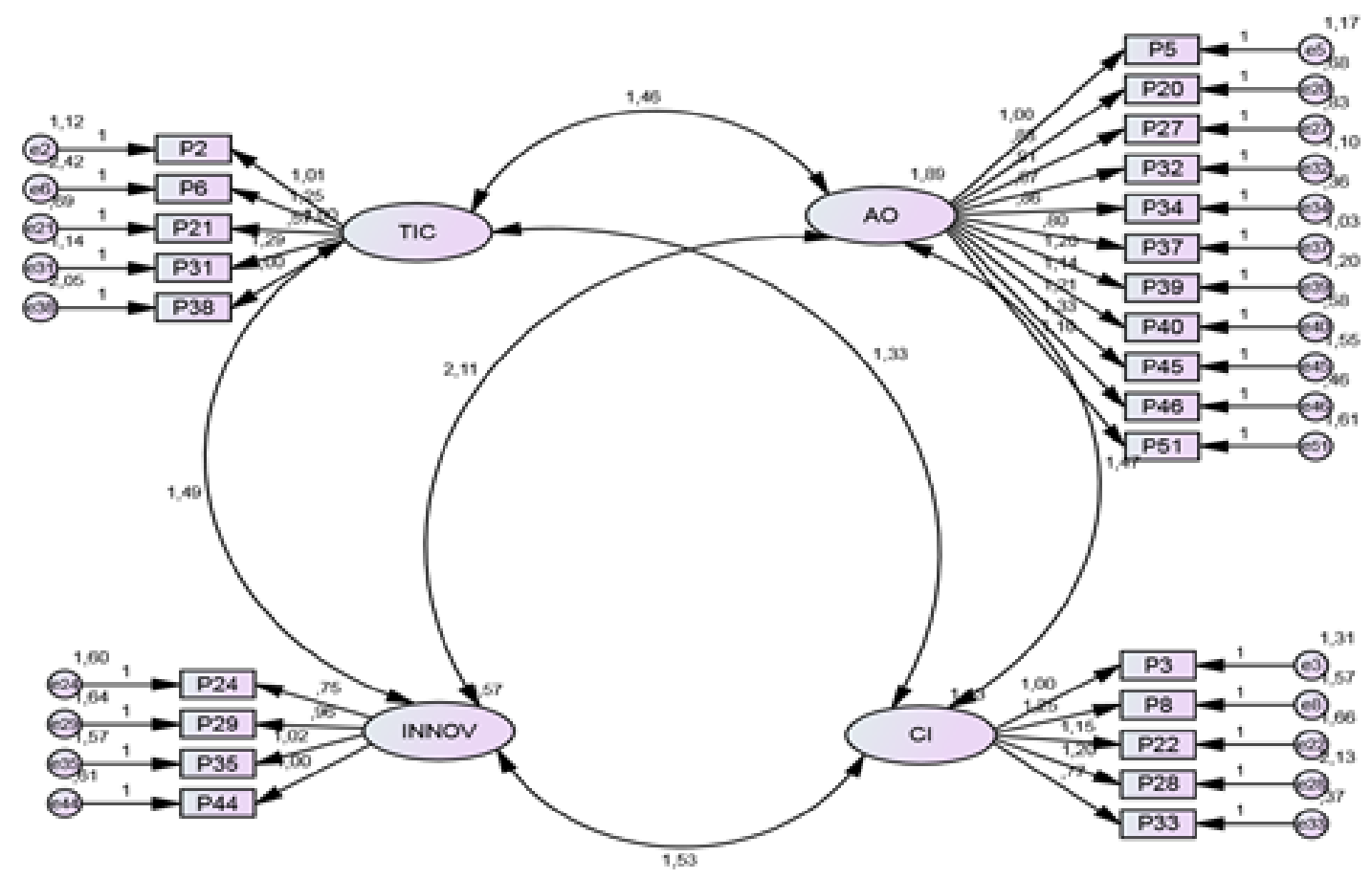

Figura 1. Modelo Inicial - Modelo Teórico.

Fuente: Datos procesados con Amos 22. Elaboración propia.

Tabla 6. Bondad de ajuste con su criterio de valor óptimo

\begin{tabular}{|c|c|c|}
\hline Estadístico & Abreviatura & Criterio \\
\hline \multicolumn{3}{|c|}{ Ajuste Absoluto } \\
\hline Chi-cuadrado & $X^{2}$ & Significación > 0.05 \\
\hline Razón Chi-cuadrado/grados de libertad & $X^{2} / g l$ & Menor que 3 \\
\hline \multicolumn{3}{|c|}{ Ajuste comparativo } \\
\hline Índice de bondad de ajuste comparativo & $\mathrm{CFI}$ & 0.95 \\
\hline Índice de Tucker-Lewis & TLI & 0.95 \\
\hline Índice de ajuste normalizado & $\mathrm{NFI}$ & 0.95 \\
\hline \multicolumn{3}{|c|}{ Ajuste parsimonioso } \\
\hline Corregido por parsimonia & PNFI & Próximo a 1 \\
\hline \multicolumn{3}{|c|}{ Otros } \\
\hline Índice de bondad de ajuste & GFI & 0.95 \\
\hline Índice de bondad de ajuste corregido & AGFI & 0.95 \\
\hline Raíz del residuo cuadrático promedio & $\mathrm{RMR}$ & Próximo a 0 \\
\hline Raíz del residuo cuadrático promedio de aproximación & RMSEA & $<0.08$ \\
\hline
\end{tabular}

Fuente: García M, (2011) 
La siguiente tabla 7, muestra las estimaciones de los valores de las covarianzas entre las variables latentes, dado que los valores $\mathrm{P}$ son menores que 0,05 estas correlaciones se consideran significativas en el modelo.

\section{Modelo Final}

Después de realizar los ajustes al modelo propuesto se obtuvo este modelo final, ver Figura 2, con su correspondiente tabla de regresión que servirá para validar las hipótesis planteadas, ver Tabla 7 e índices de ajuste que darán cuenta de la validez del modelo, ver Tabla 9.

Después de haber ajustado el modelo propuesto se observa que son óptimos los índices (CFI, IFI, RMR,
PNFI, AIC, CAIC y ECVI) y están próximos a los valores óptimos (NFI, GFI y SRM) Cabe anotar que se cumple con los valores de los indicadores para el caso de muestras pequeñas como el ECVI y con el indicador cuando los datos no presentan normalidad como el PNFI.

\section{Descripción del Modelo}

Para realizar la descripción del modelo estructural se utilizará la metodología propuesta por (Mejía y Cornejo, 2010) Escobedo et al., (2016).

ARIABLES: Consta de 4 variables latentes, 1 exógena que es TIC y 3 endógenas; $\mathrm{Cl}$, AO e INNOV. Además, tiene 11 variables superficiales u observadas, 3 de TIC, 3 de $\mathrm{AO}, 3$ de $\mathrm{Cl}$ y 2 de INNOV. Ver Tabla 7.

Tabla 7. Covarianzas Modelo Inicial - Modelo Teórico

\begin{tabular}{|c|c|c|c|c|c|c|}
\hline & & & Estimate & S.E. & C.R. & P \\
\hline $\mathrm{AO}$ & $<->$ & TIC & 1,459 &, 316 & 4,612 & $* * *$ \\
\hline $\mathrm{Cl}$ & $<->$ & INNOV & 1,533 &, 664 & 2,308 &, 021 \\
\hline $\mathrm{AO}$ & $<->$ & $\mathrm{Cl}$ & 1,472 &, 570 & 2,581 &, 010 \\
\hline $\mathrm{TIC}$ & $<->$ & INNOV & 1,494 &, 405 & 3,689 & $* * *$ \\
\hline $\mathrm{AO}$ & $<->$ & INNOV & 2,112 &, 802 & 2,632 &, 008 \\
\hline $\mathrm{Cl}$ & $<->$ & $\mathrm{TIC}$ & 1,329 &, 314 & 4,231 & $* * *$ \\
\hline
\end{tabular}

Fuente: Datos procesados con Amos 22. Elaboración propia.

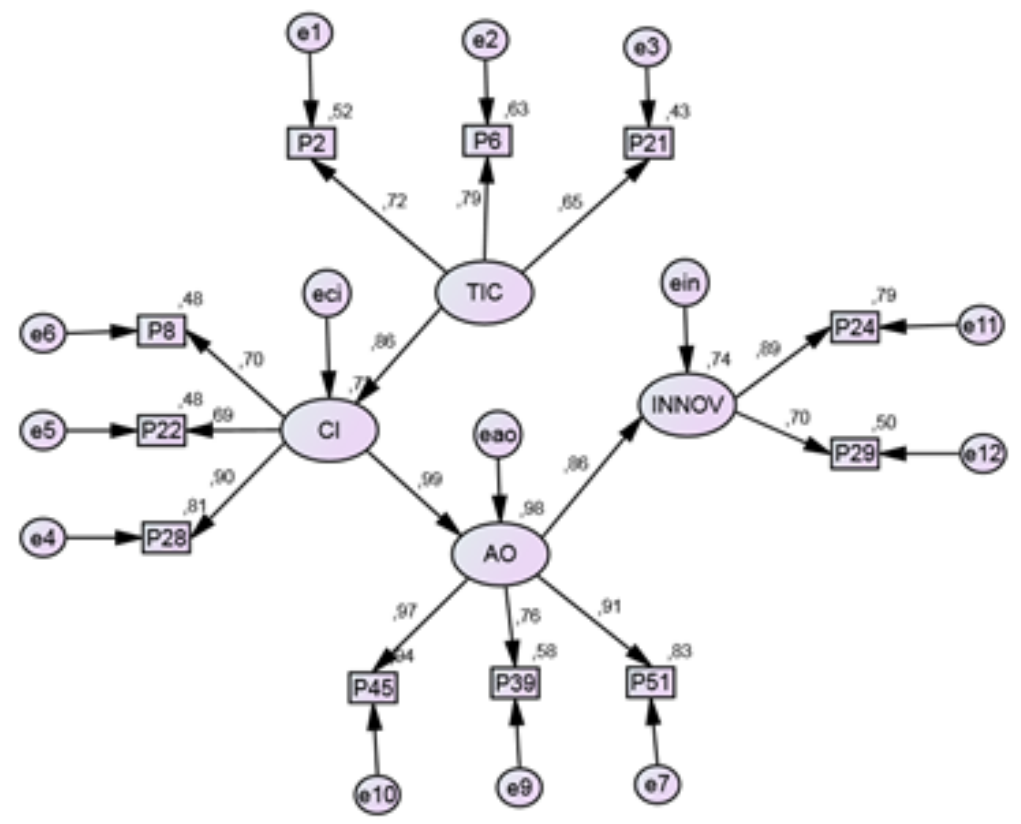

Figura 2. Modelo Estructural Final.

Fuente: Datos procesados con Amos 22. Elaboración propia. 
Tabla 8. Regresiones Modelo Final Propuesto

\begin{tabular}{|c|c|c|c|c|c|c|}
\hline & & & Estimate & S.E. & C.R. & P \\
\hline $\mathrm{Cl}$ & $<--$ & $\mathrm{TIC}$ & 2.365 & 0.832 & 2.844 & 0.004 \\
\hline $\mathrm{AO}$ & $<--$ & $\mathrm{Cl}$ & 0.951 & 0.132 & 7.79 & $* * *$ \\
\hline $\mathrm{INNOV}$ & $<--$ & $\mathrm{AO}$ & 0.830 & 0.161 & 5.160 & $* * *$ \\
\hline $\mathrm{P} 2$ & $<--$ & $\mathrm{TIC}$ & 1.000 & & & \\
\hline $\mathrm{P} 6$ & $<--$ & $\mathrm{TIC}$ & 1.749 & 0.705 & 2.481 & 0.013 \\
\hline $\mathrm{P} 21$ & $<--$ & $\mathrm{TIC}$ & 1.557 & 0.614 & 2.535 & 0.011 \\
\hline $\mathrm{P} 28$ & $<--$ & $\mathrm{Cl}$ & 1.000 & & & \\
\hline $\mathrm{P} 22$ & $<--$ & $\mathrm{Cl}$ & 0.708 & 0.174 & 4.079 & $* * *$ \\
\hline $\mathrm{P} 8$ & $<--$ & $\mathrm{Cl}$ & 0.734 & 0.181 & 4.044 & $* * *$ \\
\hline $\mathrm{P} 51$ & $<--$ & $\mathrm{AO}$ & 1.000 & & & \\
\hline $\mathrm{P} 45$ & $<--$ & $\mathrm{AO}$ & 1.116 & 0.140 & 7.962 & $* * *$ \\
\hline $\mathrm{P} 39$ & $<--$ & AO & 0.868 & 0.85 & 4.681 & $* * *$ \\
\hline $\mathrm{P} 24$ & $<--$ & $\mathrm{INNOV}$ & 1.000 & & & \\
\hline $\mathrm{P} 29$ & $<--$ & INNOV & 1.109 & 0.79 & 3.981 & $* * *$ \\
\hline
\end{tabular}

Fuente: Datos procesados con Amos 22. Elaboración propia

Tabla 9. Índices de ajuste del modelo final propuesto

\begin{tabular}{|l|l|c|c|c|}
\hline \multicolumn{1}{|c|}{ Índice } & \multicolumn{1}{c|}{ Valor óptimo } & Modelo GC & Modelo saturado & Modelo independiente \\
\hline NPAR & Escoger el mayor & 22 & 66 & 11 \\
\hline CMIN & Escoger el mayor & 53.14 & 0.0 & 223.58 \\
\hline CMIN/DF & Escoger el mayor & 1.21 & - & 4.06 \\
\hline CFI & Próximo a 1 & 0.95 & 1.0 & 0.0 \\
\hline IFI & Próximo a 1 & 0.95 & 1.0 & 0.0 \\
\hline NFI & $>0.9$ & 0.76 & 1.0 & 0.0 \\
\hline GFI & $>0.9$ & 0.70 & 1.0 & 0.21 \\
\hline AGFI & $>0.9$ & 0.54 & - & 0.06 \\
\hline RMR & Próximo a 0 & 0.30 & 0.0 & 2.42 \\
\hline SRMR & $<0.05$ & 0.086 & - & - \\
\hline PNFI & $>0.08$ & 0.61 & 0.0 & 0.0 \\
\hline RMSEA & $<0.08$ & 0.102 & - & 0.39 \\
\hline AIC & Escoger el menor & 97.14 & 132.0 & 245.58 \\
\hline CAIC & Escoger el menor & 142.12 & 266.94 & 268.07 \\
\hline ECVI & Escoger el menor & 4.86 & 6.60 & 12.28 \\
\hline
\end{tabular}

Fuente: Datos procesados con Amos 22. Elaboración propia. 


\section{Modelo Estructural}

Representa una red de relaciones en forma de un conjunto de ecuaciones lineales que enlazan variables latentes endógenas con variables latentes exógenas.
Estas son las ecuaciones (1), (2) y (3) que se muestran a continuación. ( $R_{i}$ y $r_{j}$, representan relaciones entre variables)

\begin{tabular}{|l|l|l|}
\hline $\mathrm{Cl}=\mathrm{R}_{\mathrm{TIC}-\mathrm{CI}} \mathrm{TIC}+$ eci $(1)$ & $\mathrm{AO}=\mathrm{R}_{\mathrm{Cl}-\mathrm{AO} \mathrm{Cl}+\text { eao }(2)}$ & INNOV= RAO-INNOV AO + ein (3) \\
\hline
\end{tabular}

\section{Modelo de Medida}

Especifica las variables superficiales u observadas, que van a servir para medir los conceptos teóricos propuestos en el modelo estructural. Las ecuaciones (4) a (14) especifican las variables superficiales y se muestran a continuación. Ver Tabla 10.

\section{Ajuste del Modelo de Medida}

Se analiza que tanto los conceptos teóricos del modelo están medidos a través de las variables superficiales. Para ello, lo primero a comprobar es que todas las cargas entre las variables superficiales y las variables latentes sean significativas (P-valor <0.05) Ver tabla 11 de regresión:

El modelo de medida endógeno con los valores de las cargas factoriales se muestran en las ecuaciones (4') a (14') ver Tabla 12.

\section{Ajuste del Modelo Estructural}

Al igual que se hizo con el modelo de medida, también se evalúa el modelo estructural para asegurar que todos los parámetros estimados sean significativos ( $p$ valor < 0.05) en la tabla 11 anterior, se aprecia que la relación de la variable exógena TIC, con la variable en-

Tabla 10. Modelo de Medida

\begin{tabular}{|c|c|c|}
\hline $\mathrm{P} 2=\mathrm{rP} 2-\mathrm{TIC} \mathrm{TIC}+\mathrm{e} 1(4)$ & $\mathrm{P} 6=$ rP6-TIC TIC + e2 (5) & $\mathrm{P} 21=\mathrm{rP} 21-\mathrm{TIC} \mathrm{TIC}+\mathrm{e} 3(6)$ \\
\hline $\mathrm{P} 28=\mathrm{rP}_{2} 8-\mathrm{Cl} \mathrm{Cl}+\mathrm{e} 4(7)$ & $\mathrm{P} 22=\mathrm{rP}_{22}-\mathrm{Cl} \mathrm{Cl}+\mathrm{e} 5(8)$ & $\mathrm{P} 8=$ rP8-Cl $\mathrm{Cl}+\mathrm{e} 6(9)$ \\
\hline $\mathrm{P} 51=$ rP51-AO AO + e7 (10) & $\mathrm{P} 39=$ rP39-AO AO + e9 (11) & $\mathrm{P} 45=\mathrm{rP} 45-\mathrm{AO} \mathrm{AO}+\mathrm{e} 10(12)$ \\
\hline P24= rP24-INNOV INNOV + e11 (13) & P29= rP29-INNOV INNOV + e12 (14) & \\
\hline
\end{tabular}

Fuente: Datos procesados con Amos 22. Elaboración propia.

Tabla 11. Tabla de Regresión

\begin{tabular}{|c|c|c|c|c|c|c|}
\hline & & & Estimate & S.E. & C.R. & $\mathbf{P}$ \\
\hline Capital I. & $<-$ & $\mathrm{TIC}$ & 1.567 & 0.508 & 3.084 & 0.002 \\
\hline Aprendizaje & $<-$ & Capital & 0.941 & 0.153 & 6.170 & $* * *$ \\
\hline Innovación & $<-$ & Aprendizaje & 0.867 & 0.190 & 4.574 & $* * *$ \\
\hline P2 & $<-$ & TIC & 1.000 & & & \\
\hline P6 & $<-$ & $\mathrm{TIC}$ & 1.480 & 0.469 & 3.157 & .002 \\
\hline $\mathrm{P} 21$ & $<-$ & $\mathrm{TIC}$ & 0.613 & 0.230 & 2.667 & .008 \\
\hline P28 & $<-$ & Capital & 1.000 & & & \\
\hline $\mathrm{P} 22$ & $<-$ & Capital & 0.690 & 0.182 & 3.791 & $* * *$ \\
\hline P8 & $<-$ & Capital & 0.724 & 0.189 & 3.834 & $* * *$ \\
\hline $\mathrm{P} 51$ & $<-$ & Aprendizaje & 1.000 & & & \\
\hline P39 & $<-$ & Aprendizaje & 0.856 & 0.188 & 4.546 & $* * *$ \\
\hline $\mathrm{P} 45$ & $<-$ & Aprendizaje & 1.130 & 0.138 & 8.165 & $* * *$ \\
\hline $\mathrm{P} 24$ & $<-$ & Innovación & 1.000 & & & \\
\hline P29 & $<-$ & Innovación & 0.857 & 0.238 & 3.597 & $* * *$ \\
\hline
\end{tabular}

Fuente: Datos procesados con Amos 22. Elaboración propia. 
Tabla 12. Modelo de medida endógeno con los valores de las cargas factoriales

\begin{tabular}{|c|c|c|}
\hline $\mathrm{P} 2=1.000(1.265)+1.171\left(4^{\prime}\right)$ & $\mathrm{P} 6=1.480(1.265)+1.655\left(5^{\prime}\right)$ & $\mathrm{P} 21=0.613(1.265)+0.632\left(6^{\prime}\right)$ \\
\hline $\mathrm{P} 28=1.000(0.733)+1.000\left(7^{\prime}\right)$ & $\mathrm{P} 22=0.690(0.733)+2.208\left(8^{\prime}\right)$ & $\mathrm{P} 8=0.724(0.733)+2.366\left(9^{\prime}\right)$ \\
\hline $\mathrm{P} 51=1.000(0.984)+0.793\left(10^{\prime}\right)$ & $\mathrm{P} 39=0.856(0.984)+2.016\left(11^{\prime}\right)$ & $\mathrm{P} 45=1.130(0.984)+0.319\left(12^{\prime}\right)$ \\
\hline $\mathrm{P} 24=1.000(0.741)+1.049\left(13^{\prime}\right)$ & & $\mathrm{P} 29=0.857(0.741)+2.894\left(14^{\prime}\right)$ \\
\hline
\end{tabular}

Fuente: Datos procesados con Amos 22. Elaboración propia.

dógena $\mathrm{Cl}$ y las relaciones de las variables endógenas $\mathrm{Cl}$ - AO y AO - INNOV son significativas. El modelo estructural con los parámetros estructurales se presenta a continuación en las ecuaciones $\left(1^{\prime}\right),\left(2^{\prime}\right)$ y $\left(3^{\prime}\right)$

\begin{tabular}{|c|c|c|}
\hline $\mathrm{Cl}=1.567(1.265)+1.129\left(1^{\prime}\right)$ & $\mathrm{AO}=0.941(0.733)+0.061\left(2^{\prime}\right)$ & $\mathrm{INNOV}=0.867(0.984)+1.000\left(3^{\prime}\right)$ \\
\hline
\end{tabular}

\section{Discusión}

El instrumento diseñado es confiable según los resultados de la prueba Alfa de Cronbach y útil para medir la Gestión del Conocimiento del Departamento de Sistemas y Recursos Tecnológicos de la universidad, lo que confirma lo recomendado por Rodrigo A. (2006) en el sentido de utilizar datos primarios e instrumentos previamente validados, para garantizar la calidad de la investigación.

Las variables del modelo teórico propuesto, según el tratamiento estadístico: Aprendizaje Organizacional, TIC, Capital Intelectual e Innovación, que conforman el modelo de los datos resultado de la aplicación del instrumento utilizando ecuaciones estructurales, nos permitió confirmar la teoría, dado que están altamente correlacionadas. Este resultado confirma lo planteado por Cegarra, Alonso y Monreal (2006); Garzón y Fischer (2010) muestran que el capital estructural promueve el flujo de conocimiento $y$, acompañado de innovación, mejora la gestión de los procesos de negocio al hacer uso de las TIC; estas también apoyan el aprendizaje organizacional y el capital intelectual que, aunados a una cultura y condiciones que permitan su desarrollo, son claves para lograr organizaciones sustentables (Garzón y Fisher, 2008).

El uso de las (TIC) produce un efecto positivo en el Capital Intelectual $(\mathrm{CI})$ del Departamento de Sistemas y Recursos Tecnológicos de la universidad. Se confirma que el uso de las TIC mejora la gestión de los procesos, es un catalizador del flujo de conocimiento y si se utiliza de manera transversal en todos los procesos de la organización se convierte en un factor de éxito. El dominio de las TIC es la razón de ser del Departamento de Sistemas y Recursos Tecnológicos de la Universidad. Las TIC hacen parte del capital estructural de la organización y constituyen la única variable exógena del modelo propuesto, representado por herramientas de comunicación y colaboración, al igual que por métodos y técnicas que dinamizan los procesos. En el capital estructural deben confluir los conocimientos aportados por el capital humano y el relacional, convirtiéndose en el valor agregado de la organización, que la diferencie en el mercado (Cegarra, Alonso y Monreal, 2006)

El Capital Intelectual $(\mathrm{Cl})$ produce un efecto positivo en el Aprendizaje Organizacional (AO) del Departamento de Sistemas y Recursos Tecnológicos de la universidad. Se valida lo planteado por Tseng (2008) y O'Brien y Marakas (2006), cuando afirman que el capital intelectual, en nuestro modelo representado por el capital estructural, específicamente las TIC; facilitan las actividades realizadas por todos los stakeholders, ayudan a gestionar el aprendizaje y mejorar la manera en que compiten las organizaciones.

El Aprendizaje Organizacional (AO) impacta positivamente la Innovación (INNOV) en el Departamento de Sistemas y Recursos Tecnológicos de la universidad. En sintonía con lo expuesto por Nelson y Winter (1982); Manual de Oslo (2006) y Rodríguez (2006); Los procesos de innovación son dinámicos, asociados al conocimiento tácito y explícito, que les permiten a las organizaciones resolver sus problemas implican cambios en las prácticas empresariales y que además requieren que exista una cultura y un clima que estimule la creatividad.

\section{Conclusiones}

Después de haber realizado el análisis estadístico aplicando las técnicas descriptivas, combinadas con el análisis factorial, los SEM (Modelos de Ecuaciones Estructurales), utilizando los programas estadísticos SPSS 
25, AMOS 22 y STATGRAPHICS Centurión XVI, y haber interpretado los resultados obtenidos en cada análisis se tienen las siguientes conclusiones:

Después de una exhaustica revisión de la teoría dominante sobre la gestión del conocimiento, y de haber contrastado los resultados de la investigación llevada a cabo; se determinó que para el Departamento de sistemas y recurso tecnológico de la Universidad Simón Bolívar de la ciudad de Barranquilla; las variables más influyentes en la gestión del conocimiento son:

- Las TIC, principalmente en lo referente a los servicios principales y de infraestructura.

- El capital intelectual, enfocado al capital estructural en lo referente al clima organizacional y los procesos de negocios.

- El aprendizaje organizacional, distinguiéndose el Sistema cultural y el aprendizaje en equipo.

- La innovación, básicamente referida a políticas que motiven la creatividad del personal.

Diseñar un instrumento de medición al momento de realizar una investigación es muy ventajoso porque los datos obtenidos corresponden específicamente a las variables analizadas en la fase teórica, situación que no siempre se consigue al utilizar instrumentos realizados para otras investigaciones u otras poblaciones, que en algunos casos exige adaptar el instrumento y volver a validarlo, lo que requiere una considerable carga de trabajo adicional.

Hipótesis 1: El instrumento diseñado es confiable según los resultados de la prueba Alfa de Cronbach. Ver Tabla 4, mediante el análisis factorial de componentes principales y el método de rotación Varimax se eliminaron los 140 ítems que menos influían sobre las variables latentes, con el fin de que cumplieran los criterios de unidimensionalidad, varianza mayor al $60 \%$ y las puntuaciones superiores al 50\% (0.5) Mediante el análisis factorial confirmatorio utilizando las ecuaciones estructurales SEM se puede afirmar que el modelo propuesto de Gestión del Conocimiento conformado por las variables Aprendizaje Organizacional, TIC, Capital Intelectual e Innovación; es empíricamente confiable y el instrumento diseñado puede ser utilizado para medir este constructo. Ver Tabla 7.

Hipótesis 2: El uso de las (TIC) produce un efecto positivo en el Capital Intelectual (Cl) del Departamento de Sistemas y Recursos Tecnológicos de la Universidad Simón Bolívar sede Barranquilla.
Según el p-valor de la tabla de regresiones del modelo final propuesto $\mathrm{Cl} \leftarrow \mathrm{TIC}=0,004$. Por tanto, se rechaza la hipótesis nula. Ver tabla 8.

Hipótesis 3: El Capital Intelectual $(\mathrm{Cl})$ produce un efecto positivo en el Aprendizaje Organizacional (AO) del Departamento de Sistemas y Recursos Tecnológicos de la Universidad. Según el p-valor de la tabla 8 de regresiones del modelo final propuesto $\mathrm{AO} \leftarrow \mathrm{Cl}=0,0$. Por lo tanto, se rechaza la hipótesis nula.

Hipótesis 4: El Aprendizaje Organizacional (AO) impacta positivamente la Innovación (INNOV) en el Departamento de Sistemas y Recursos Tecnológicos de la Universidad Simón Bolívar sede Barranquilla. Según el p-valor de la tabla 8 de regresiones del modelo final propuesto INNOV $\leftarrow A O=0,0$. Por lo tanto, se rechaza la hipótesis nula.

Hipótesis 5: De acuerdo con los p-valores relacionados en la tabla 7 de covarianzas del modelo; $\mathrm{AO} \leftarrow \rightarrow \mathrm{TIC}=0,0 ; \quad \mathrm{Cl} \leftrightarrow \mathrm{INNOV}=0,021 ; \quad \mathrm{AO} \leftarrow \rightarrow \mathrm{Cl}=$ 0,$010 ; \mathrm{TIC} \leftrightarrow \mathrm{INNOV}=0,0 ; \mathrm{AO} \leftarrow \rightarrow I N N O V=0,008 ;$ $\mathrm{Cl} \leftarrow \mathrm{TIC}=0,0$. Por lo tanto se rechaza la hipótesis nula.

El modelo propuesto evidencia la multidimensionalidad de la gestión del conocimiento, ver Tabla 7; siendo los indicadores más relevantes por dimensión:

Las TIC, principalmente en lo referente a los servicios principales y de infraestructura. El capital intelectual, enfocado al capital estructural en lo referente al clima organizacional y los procesos de negocios. El aprendizaje organizacional, distinguiéndose el Sistema cultural y el aprendizaje en equipo. La innovación, básicamente referida a políticas que motiven la creatividad del personal.

Mediante la técnica de modelos de ecuaciones estructurales SEM se comprobaron las hipótesis estadísticas planteadas con una confianza del 95\%, con ello se evidenciaron las relaciones causales entre las variables latentes y se ajustó el modelo para representar la gestión del conocimiento del Dpto. de sistemas y recurso tecnológico de la Universidad Simón Bolívar sede Barranquilla.

Después de ajustar el modelo propuesto que explica más del $70 \%$ de la varianza del capital intelectual, el aprendizaje organizacional y la innovación del Departamento de Sistemas de la Universidad Simón Bolívar sede Barranquilla queda de manifiesto que el resultado de una adecuada gestión del conocimiento se deberá ver reflejada en el grado de innovación logrado. 


\section{Implicaciones directivas}

Se recomienda a los responsable de la gestión del Departamento de Sistemas y Recursos Tecnológicos de la Universidad intervenir los indicadores más relevantes de la dimensión Aprendizaje Organizacional (El Sistema cultural y El Aprendizaje en equipo), TIC (Servicios Principales y Servicios de Infraestructura), Capital Intelectual (Clima Organizacional y Procesos) e Innovación (Motivadores y Generadores de Ideas) ya que las iniciativas que los involucren deberían tener un impacto notorio en la gestión del conocimiento que se podría evidenciar en mejoras de las condiciones de las variables que conforman el modelo y que en últimas debería reflejarse en los procesos de innovación del departamento.

\section{Limitaciones del Estudio}

Aunque los datos analizados no cumplieron el criterio de normalidad (prueba K-S; Kolmogorov Smirnov) ya que el P-valor es menor al nivel de significancia de 0,05 , ver tabla 5 y el tamaño de la muestra es relativamente pequeño con solo 21 casos que son los integrantes del Departamento de Sistemas y Recursos Tecnológicos de la Universidad; se aplicó la técnica de los modelos estructurales SEM con el software estadístico Amos IBM SPSS 25. Para ajustar los modelos basándose en los indicadores para muestra pequeña ECIV y para la falta de normalidad el indicador PNFI, además se realizó un análisis con esta metodología para mejorar los indicadores GFI, AGFI, RMR y SRMR. Los cuales se ajustaron hasta un valor cercano al valor óptimo de estos indicadores.

\section{Referencias}

Abreu J. Constructos, Variables, Dimensiones, Indicadores y Congruencia (Constructs, Variables, Dimensions, Indicators y Consistency). Daena, 2012;7(3):123-130.

Alvarado L. (2009) Sistema de gestión del conocimiento programa de textos escolares, Proyecto de grado para optar al grado de magister en Ingeniería de negocios con tecnologías de información, Universidad De Chile; Facultad De Ciencias físicas y matemáticas; Departamento De ingeniería Industrial, Santiago.

Alvarado M, Alvarado L, Burgos E. (2012) Gestión del conocimiento en tecnología de la información: Análisis de la experiencia en el Sistema de Bibliotecas de la UC. Serie Bibliotecología y Gestión de Información, n. 70. [JournalArticle (On-line/Unpaginated)]

Andriessen D. "IC valuation and measurement: Classifying the state of the art" in Journal of Intellectual Capital, 2004;5(2): 230-242.

Argyrous G. (2011) Statistics for Research. SAGE Publications Ltd.

Asún R. (2006) Construcción de cuestionarios y escalas: El proceso de la producción de información cuantitativa; Metodologías de investigación 1로 ed. - Santiago: Lom Ediciones.
Barragán A. Aproximación a una taxonomía de modelos de Gestión del Conocimiento. Intangible Capital, 2009;5(1):65-101.

Batista-Foguet JM, Coenders G, Alonso J. Análisis factorial confirmatorio. Su utilidad en la validación de cuestionarios relacionados con la salud, Med Clin (Barc); 2004;122(Supl 1):21-27.

Bernal J, de Nieves C, Briones A. Aplicación de un modelo de ecuaciones estructurales para analizar los sistemas de gestión en la integración de la RSC y su influencia en la estrategia y el performance de las empresas tecnológicas // Applying a Structural Equation Model to Analyze Management Systems in the Integration of CSR and its Influence on the Strategy and Performance of Technology Companies. Rev Métodos Cuant. Econ Empresa, 2016;21:77-102. Recoopered de https://www.upo. es/revistas/index.php/RevMetCuant/article/view/2254

Bontis N, Keow WCC, Richardson S. "Intellectual capital and business performance in Malaysian industries". Journal of Intellectual Capital, 2000;1(1):85-100.

Bueno E, Salmador M, Merino C. Génesis, concepto y desarrollo del capital intelectual en la economía del conocimiento: Una reflexión sobre el Modelo Intellectus y sus aplicaciones. Estudios de Economía Aplicada, 2008;26:43-63.

Bukowitz WR, Williams RL. (1999) The Knowledge Management Fieldbook UpperSaddle River, N.J.: Financial Times, Prentice Hall.

Cabezas-González M, Casillas-Martín S, Sanches-Ferreira M, Teixeira-Diogo F. Validación de un instrumento para medir la competencia digital de estudiantes universitarios (CODIEU). Revista de Estudios e Investigación en Psicología y Educación, 2017;13:021024. doi: http://dx.doi.org/10.17979/reipe.2017.0.13.2180

Camargo H, Rengifo P, Serrato S. (2006) Una propuesta para estructurar la gestión del conocimiento en una pyme. Fundación Universitaria Konrad Lorenz.

Cazau P. (2006). Introducción a la Investigación en Ciencias Sociales. Tercera Edición. Buenos Aires, Módulo 404 Red de Psicología online - www.galeon.com/pcazau

Cegarra JG, Alonso J, Monreal GAF. Influencia del uso de las tecnologías de internet en el capital estructural. Investigaciones Europeas de Dirección y Economía de la Empresa (IEDEE), 2006;12(3):217-228.

Choo CW, Bontis N. (2002): The strategic management of intellectual capital and organizational knowledge, NY: Oxford University Press, New York

Cupani M. Análisis de Ecuaciones Estructurales: conceptos, etapas de desarrollo y un ejemplo de aplicación, Revista Tesis. 2012;1:186-199.

Dávila M, Coronado J, Cerecer B. Las dimensiones de la calidad del servicio en el proceso de distribución y comercialización de energía eléctrica. Contaduría y Administración, 2012;57(3):175195.

De Vita N. (2008) Tecnologías de información y comunicación para las organizaciones del siglo XXI. Centro de Investigación de Ciencias Administrativas y Gerenciales. Instituto Universitario de Tecnología de Maracaibo. ISSN: 1856-6189. Volumen 5 Edición No. 1 
Drucker P. (2000) Gestión del Conocimiento: Llega una nueva organización a la empresa. Harvard Business Review. Bilbao.

Escobedo M, Hernández-Gómez JA, Estebané-Ortega V, MartínezMoreno G. Modelos de ecuaciones estructurales: Características, fases, construcción, aplicación y resultados. Ciencia y trabajo, 2016;18(55):16-22. https://dx.doi.org/10.4067/S071824492016000100004

FUTURE TRENDS FORUM. (2007): Innovation: The wealth of nations. Resumen Ejecutivo, Fundación de la Innovación Bankinter, Madrid.

García M. (2011a) Proyecto de grado Análisis Causal con Ecuaciones Estructurales de la Satisfacción Ciudadana con los Servicios Municipales. Universidad Santiago de Compostela, España

García M. (2011b) Proyecto de grado Análisis Causal con Ecuaciones Estructurales de la Satisfacción Ciudadana con los Servicios Municipales. Universidad Santiago de Compostela, España

Garvin D. Building a Learning Organization. Harvard Business Review, 1993;71(4):78-91.

Garzón MA, Fisher AL. Modelo teórico de aprendizaje organizacional. Pensamiento y Gestión, 2008;24:195-224.

Gómez J, Villarreal M, Villarreal F. Las incapacidades de aprendizaje organizacional y su relación con los estilos de aprendizaje organizacional en la Mipyme. Contaduría Y Administración, 2015;60(3):686-702. Doi: http://dx.doi. org/10.1016/i.cya.2013.11.001

Guba EG, Lincoln YS. (1981). Effective evaluation. San Francisco, CA: Jossey-Bass.

Haenlein M, Kaplan A. A beginner's guide to partial least squares analysis. Und statistics, 2004;3(4):283-297.

Hamel G, Prahalad CK. "Intention Estratégica". Harvard Business Review, 1989;67(3):63.

Housel T, Bell A. (2001) Measuring and Managing Knowledge. Mc Graw-Hill Higher Education.

Hoyos et al., (2009). "Personas y Cultura Social Innovadora: Propuesta de un modelo explicativo": En Cossío, F.J. (Eds): Administrando en entornos inciertos - XXIII Congreso Anual AEDEM, ESIC Editorial, Madrid. (Publicación en Formato Electrónico).

Jöreskog KG, Sörbom D. Advances in factor analysis and structural equation models. Cambridge (MA): developments in structural equation modeling. J Mark. Res, 1982;19:404-416.

Jöreskog KG. (1974). Analyzing psychological data by structural analysis of covariance matrices. Contemporary developments in mathematical psychology. S. Francisco (CA): Freeman.

Jöreskog KG. (1979a). Statistical Estimation of Structural, Models in Longitudinal Developmental Investigations. In J.R. Nesselroade y P.B. Baltes. Longitudinal research in the study of the behavior and development. New York: Academic Press.

Jöreskog KG. (1979b). A general approach to confirmatory maximum likelihood factor analysis with addendum. In K.G.
Kalpič B, Bernus P. Business process modelling through the knowledge Management perspective. I Knowl Manag, 2006;10(3):40-56.

Kaplan D. (2000). Structural equation modeling: Foundations and extensions. Newbury Park, CA: Sage.

Klasson K. Managing knowledge for advantage. The Cambridge Information Network Journal, 1999;1(1):33-41.

Kline RB. (2005). Principles and practice of structural equation modeling (2nd Ed.). New York: Guilford.

León Carrascosa V, Fernández-Díaz M. Diseño y validación de un instrumento para evaluar la participación de las familias en los centros educativos//Design and validation of an instrument for assessment the involvement of families in schools. Revista Española de Orientación y Psicopedagogía-REOP, 2018;28(3):115132.

Lincoln Y, Guba E. (1985), Naturalistic Inquiry, Bervely Hills, Sage publications

Lozano S, Rodríguez-Mejía F. (2012). Katheryne. Modelo para la implementación de ITIL en una institución universitaria. Santiago de Cali, 2012, 123p.Tésis Maestría (Magíster en Gestión de Informática y Telecomunicaciones concentración en Gerencia de Tecnología de Información y Comunicaciones). Universidad Icesi. Facultad de Ingeniería.

Maier R. (2007). Knowledge Management Systems: Information and Communication Technologies for Knowledge Management. $3^{\text {a }}$ ed. Berlin: Springer-Verlag.

Mejía M, Cornejo C. Aplicación del modelo de ecuaciones estructurales a la gestión del conocimiento. LACJEE, 2010;4(1):23-30.

Nelson R, Winter S. (1982), An Evolutionary Theory of Economic Change. Belknap Press/Harvard University Press: Cambridge.

Nonaka I, Takeuchi H. (1999). La organización creadora de conocimiento. Cómo las compañías japonesas crean la dinámica de la innovación. Trad. Martín Hernández Kocka. México: Oxford UniversityPress.

Nunnally JC, Bernstein IJ. (1995). Teoría psicométrica. Madrid: MCGraw-Hill.

O'brien JA, Marakas GM. (2006): Sistemas de información gerencial. $7^{\text {a }}$ Edición. México: McGraw-Hill.

Ocampo S, Moreno-Laverde R, Caicedo SM. Implementación de modelo de procesos de gestión de servicios con itil (information technology infrastructure library). Scientia Et Technica, 2009;41(1):215-220.

Prieto I. (2003). Una Valorización de la Gestión del Conocimiento para el Desarrollo de la Capacidad de Aprendizaje en las Organizaciones: Propuesta de un Modelo. Tesis doctoral, Universidad de Valladolid.

Rodríguez Gómez, D. (2006). Modelos para la creación y gestión del conocimiento: Una aproximación teórica. Educar, 37:2539. 
Rodríguez-Castellanos A, Hagemeister M, Ranguelov S . Absorptive capacity for R\&D: The identification of different firm profiles. Eur Plan Stud, 2010;18(8):1267-1283.

Salcines-Talledo I, González-Fernández N. Los Smartphones en Educación Superior. Diseño Y Validación De Dos Instrumentos De Recogida De Información sobre la Visión del Alumnado. Revista Española de Orientación y Psicopedagogía (REOP), 2015;26(3):96-120.

Schulze A. (2003). Knowledge Management in Innovation Processes. In G. H. Max von Zedtwitz, Tarek M. Khalil, Louis A Lefebre (Ed.), Management of Technology: Growth through Business Innovation and Entrepreneurship. Amsterdam: Pergamon.

Seaton C, Breso S. El desarrollo de un sistema de gestión del conocimiento para los institutos tecnológicos. Espacios. [online]. set. 2001;22(3):29-46. [citado 05 diciembre 2012], Disponible en la World Wide Web:<http://www.scielo.org.ve/scielo. php?script=sci_arttext\&pid=S0798-10152001000300004\&lng $=e s \& n r m=i s o>$. ISSN 0798-1015.

Thompson A, Strickland A. (2004). Administración Estratégica. Editorial Mc Graw Hill, México.
Tiwana A. (2000). The knowledge management toolkit: practical techniques for building a knowledge management system. Prentice Hall PTR.

TSENG, Shu-Mei. The effects of information technology on knowledge management systems, Expert Syst Appl, 2008;35(1-2):150160. ISSN 0957-4174, 10.1016/j.eswa.2007.06.011.

Wright S. Correlation and causation. J Agric Res, 1921;20:557-585.

Wright S. The method of path coefficients. Ann Math Stat, $1934 ; 5: 161-215$

Zabaleta de Armas M, Brito-Carrillo LE, Garzón-Castrillón MA. Modelo de Gestión del Conocimiento en el Área de TIC para una Universidad del Caribe Colombiano. Rev Lasallista Investig, 2016;13(2):136-150.

Zack MH. Developing a Knowledge Strategy. Calif Manag Rev, 1999;41(3):125-145. Disponible en: http://web.cba.neu. edu/ mzack/articles/kstrat/kstrat.htm

Milena Zabaleta:https://orcid.org/0000-0001-7890-2838 Luís Brito: https://orcid.org/0000-0002-9212-0805 Manuel Garzón: https://orcid.org/0000-0001-9009-3324 